\title{
O Caderno CRH agradece o apoio dos seguintes consultores que emitiram pareceres dos artigos para publicação na revista no ano 2018
}

Adelia Miglievich

Adriana da Silva Vinholi Rampazo

Adriano Premebida

Aline Suelen Pires

Alvaro Augusto de Borba Barreto

Andrea Maria Silveira

Angela Randolpho Paiva

Angélica Lovatto

Arnaldo Nogueira

Bárbara Castro

Carla Cecilia Ferreira

Carlos Freitas

Carlos Machado

Cinara L. Rosenfield

Claudia Rodrigues de Freitas

Delma Pessanha Neves

Denilson Bandeira Coêlho

Antonio Dimas Cardoso

Edgard Porto

Fabricio Maciel

Fátima Cabral

Flavio Mendes

Fréderic Vandenberghe

Giovanni Alves

Glaucia Villas Bôas

Luiz Gustavo da Cunha de Souza

Henrique Amorim

Jacob Carlos Lima

Javier Alberto Vadell

Joana Aparecida Coutinho

José Ricardo Ramalho

Josué Pereira da Silva

Lívia Moraes

Lucio Flavio de Almeida

Ludmila Mendonça Lopes Ribeiro

Luiz Alex Saraiva

Luiz Eduardo Pereira da Motta

Luiz Filgueiras

Luiz Martinez

Madalena Gracioli

Marcelo Badaró
Universidade Federal do Espírito Santo

Universidade Estadual de Londrina

Universidade Federal do Rio Grande do Sul

Universidade Federal de São Carlos

Universidade Federal de Pelotas

Universidade Federal de Minas Gerais

Pontifícia Universidade Católica do Rio de Janeiro

Universidade Estadual Paulista Júlio de Mesquita Filho

Universidade de São Paulo

Universidade Estadual de Campinas

Universidade Federal do Rio de Janeiro

Univerisdade Federal da Bahia

Universidade de Brasília

Universidade Federal do Rio Grande do Sul

Universidade Federal do Rio Grande do Sul

Universidade Federal Fluminense

Universidade de Brasília

Universidade Estadual de Montes Claros

Superintendência de Estudos Econômicos e Sociais da Bahia

Universidade Federal Fluminense

Universidade Estadual Paulista Júlio de Mesquita Filho

Universidade Estadual de Campinas

Universidade Federal do Rio de Janeiro

Universidade Estadual Paulista Júlio de Mesquita Filho

Universidade Federal do Rio de Janeiro

Universidade Federal de Santa Catarina

Universidade Federal de São Paulo

Universidade Federal de São Carlos

Pontifícia Universidade Católica de Minas Gerais

Universidade Federal do Maranhão

Universidade Federal do Rio de Janeiro

Universidade Estadual de Campinas

Universidade Federal do Espírito Santo

Pontifícia Universidade Católica de São Paulo

Universidade Federal de Minas Gerais

Universidade Federal de Minas Gerais

Universidade Federal do Rio de Janeiro

Universidade Federal da Bahia

Fondation Maison des sciences de L'Homme

Universidade Estadual Paulista Júlio de Mesquita Filho

Universidade Federal Fluminense 


\author{
Marcelo Ridenti \\ Marcelo Weishaupt Proni \\ Marcia Grisotti \\ Marcia Lima \\ Maria Aparecida da Cruz Bridi \\ Maria Orlanda \\ Marilia Sposito \\ Mario Henrique Guedes Ladosky \\ Marisa Silva Amaral \\ Marjorie Correa Marone \\ Milton Lahuerta \\ Monika Dowbor \\ Niemeyer Almeida Filho \\ Patricia Lanes Araujo de Souza \\ Patricia Villen \\ Paula Marcelino \\ Renato Paula \\ Roberta Traspadini \\ Roberto Goulart Menezes \\ Rodrigo de Souza Dantas Mendonça \\ Rubens Sawaya \\ Sarah da Silva Telles \\ Sávio Cavalcanti \\ Silvia Beatriz Adoue \\ Silvio Camargo \\ Sonia Maria Karam Guimarães \\ Tataiane Araujo \\ Thula Pires \\ Ximena Simpson
}

Universidade Estadual de Campinas

Universidade Estadual de Campinas

Universidade Federal de Santa Catarina

Universidade de São Paulo

Universidade Federaldo Paraná

Universidade Estadual Paulista Júlio de Mesquita Filho

Universidade de São Paulo

Universidade Federal de Campina Grande

Universidae Federal de Uberlândia

Universidae Federal de Minas Gerais

Universidade Estadual Paulista Júlio de Mesquita Filho

Universidade do Vale do Rio dos Sinos

Universidade Federal de Uberlândia

Universidade Estadual do Rio de Janeiro

Universidade Estadual de Campinas

Universidade de São Paulo

Universidade Federal de Goiás

Universidade Federal de Santa Catarina

Universidade de Brasília

Universidade de Brasília

Pontifícia Universidade Católica de São Paulo

Pontifícia Universidade Católica de Rio de Janeiro

Universidade Estadual de Campinas

Universidade Estadual Paulista Júlio de Mesquita Filho

Universidade Estadual de Campinas

Universidade Federal do Rio Grande do Sul

Universidade Federal da Bahia

Pontifícia Universidade Católica de Rio de Janeiro

Universidad Nacional de San Martin 